Revista

Actualidades Investigativas

en Educación

\title{
Tecnologías del habla para la educación inclusiva
}

Speech technologies for inclusive education

\author{
Volumen 20, Número 1 \\ Enero - Abril \\ pp. 1-24
}

\author{
Marvin Coto Jiménez \\ Maribel Morales Rodríguez
}

\section{Citar este documento según modelo APA}

Coto Jiménez, Marvin y Morales Rodríguez, Maribel. (2020). Tecnologías del habla para la educación inclusiva. Revista Actualidades Investigativas en Educación, 20(1), 1-24. Doi. 10.15517/aie.v20i1.40129 


\title{
Tecnologías del habla para la educación inclusiva
}

\author{
Tecnologías del habla para la educación inclusiva
}

\section{Marvin Coto Jiménez 1 Maribel Morales Rodríguez ${ }^{2}$}

\begin{abstract}
Resumen: Existen condiciones de discapacidad que comprometen la habilidad de las personas para expresarse utilizando su voz. Esto puede constituir una limitante para la comunicación, y una barrera para la inclusión de estudiantes con estas condiciones en contextos educativos tradicionales, donde la comunicación verbal es predominante. Dado que la educación es un derecho humano, es imperativo establecer opciones para que el estudiantado con discapacidad sea incluido en sistemas educativos donde pueda ejercer plenamente sus derechos, lo que ha llevado a la investigación, análisis y creación de sistemas de comunicación aumentativos y alternativos. En este ensayo se presentan el tema de tecnologías del habla, las cuales tienen como finalidad replicar la capacidad humana de comunicarse por esta vía apoyándose en dispositivos electrónicos, y el potencial de aplicación de estas tecnologías en los procesos de educación inclusiva. Para esto se da una visión general de los conceptos teóricos, las tendencias y los puntos de encuentro de la educación inclusiva y la tecnología, que hacen factible la creación de sistemas y dispositivos que impacten positivamente la calidad de vida del alumnado en su desarrollo educativo. Para que estas posibilidades tecnológicas sean una realidad en las aulas y los diversos entornos educativos, se propone el establecimiento de equipos interdisciplinarios amplios que permitan un abordaje integral de cada caso particular. De esta manera, las soluciones se pueden convertir en herramientas concretas verdaderamente útiles y confiables para el usuario, su familia y su entorno educativo.
\end{abstract}

Palabras clave: derechos humanos, discapacidad, acceso a la educación, tecnología.

\begin{abstract}
There are conditions of disability that compromise the ability of people to express themselves using their voice. This may constitute a limitation for communication, and a barrier to the inclusion of students with these conditions in traditional educational contexts, where verbal communication is predominant. Since education is a human right, it is imperative to establish options for students with disabilities to be included in educational systems where they can fully exercise their rights, which has led to the research, analysis and creation of augmentative and alternative communication systems. This essay presents the most recent developments in speech technologies, which are intended to replicate the human capacity to communicate in this way based on electronic devices, and the potential for application of these technologies in the processes of educational inclusion. We give an overview of the theoretical concepts, trends and meeting points of educational inclusion and technology, which make it possible to create systems and devices that positively impact the quality of life of students in their educational development. To make these technological a reality in the classrooms and other educational environments, it is proposed the establishment of broad interdisciplinary teams that allow an approach integral of each particular case. In this way, solutions can become concrete tools that are truly useful and reliable for the user, his family, and his educational environment.
\end{abstract}

Keywords: human rights, disability, access to education, technology.

\footnotetext{
${ }^{1}$ Universidad de Costa Rica, Costa Rica. Dirección electrónica: marvin.coto@ucr.ac.cr. ORCID: https://orcid.org/0000-0002-6833-9938

2Universidad de Costa Rica, Costa Rica. Dirección electrónica: maribel.moralesrodriguez@ucr.ac.cr
}

Ensayo recibido: 28 de junio, 2019

Enviado a corrección: 24 de setiembre, 2019

Aprobado: 14 de octubre, 2019 


\section{Introducción}

En Costa Rica el proceso de educación inclusiva ha constituido un reto permanente. A lo largo de la historia este proceso ha enfrentado barreras actitudinales y procedimentales en las aulas de todos los niveles educativos. No obstante, existe en nuestros días un marco normativo e institucional con el cual se apuesta por este enfoque como la vía idónea para la construcción de una sociedad más equitativa y solidaria, tanto a nivel internacional dentro de la agenda 2030 de la Organización de Naciones Unidas (ONU) como a nivel nacional con la publicación el año 2018 del decreto ejecutivo № 40955 del Ministerio de Educación Pública de Costa Rica (MEP) en el que se establece como norma nacional la inclusión y la accesibilidad al sistema educativo costarricense.

A pesar de esto, cuando se trata de educación inclusiva para la población en condición de discapacidad, existen aún barreras complejas debido a que el cuerpo docente se enfrenta a carencias en la formación, herramientas, técnicas, estrategias y conceptualización de las manifestaciones conductuales, sensoriales, cognitivas, de movilidad o comunicativas propias de la diversidad humana, que son relevantes en el contexto de aula para poder mediar el proceso de enseñanza desde la propuesta del Diseño Universal del Aprendizaje (DUA).

EI DUA nace en la década de los años noventa, cuando la idea del diseño universal que aboga por el acceso a infraestructura, servicios y productos se traslada al marco educativo. En éste, se propone un cambio de enfoque para los procesos pedagógicos en donde se considera fundamental "los avances en el diseño arquitectónico, la evolución de las tecnologías de la educación y los resultados de las investigaciones sobre el cerebro" (AlbaPastor, 2012 pp.3), para garantizar el acceso de los niños, niñas y adolescentes al currículo educativo.

En Costa Rica, durante la última década, se ha venido fortaleciendo la oferta educativa con una nueva propuesta que ha tomado acciones como la consigna de una educación para todos y la transformación de los servicios de apoyos para el estudiantado en condición de discapacidad. Estas acciones a su vez se sustentan en uno de los elementos del DUA, que ofrece una visión del quehacer pedagógico teniendo como base el neurodesarrollo y las redes neurales que se construyen durante el mismo, para hacer frente, entre otros aspectos fundamentales de la vida, a la adquisición del aprendizaje. 
EI DUA propone tres redes de aprendizaje a las que denomina de reconocimiento, estratégica y afectiva, las cuales dan respuesta al qué, cómo y por qué se aprende, mediante "tres principios fundamentales en la aplicación de este modelo a la enseñanza, a los que se asocian una serie de pautas para su aplicación en la práctica educativa:

- Proporcionar múltiples medios de representación.

- Proporcionar múltiples medios de acción y expresión.

- Proporcionar múltiples formas de implicación”. (Alba-Pastor, 2012 pp.4)

Es ante estas pautas que surgen algunos cuestionamientos de cómo el estudiantado en condición de discapacidad puede ejercer su derecho a un aprendizaje en el que se le garantice acceder a la información, por el medio o los medios de representación idóneos para sus formas y habilidades comunicativas. Adicionalmente el cómo se les garantiza la expresión de la información por diferentes alternativas de comunicación y finalmente el cómo lograr una vinculación con el proceso de aprendizaje desde la motivación, que mantenga el interés en las acciones pedagógicas y de aprendizaje.

Conviene aclarar que es a partir de la reflexión sobre los cuestionamientos anteriores donde el uso de las tecnologías propone una variabilidad de oportunidades para fomentar la educación inclusiva en donde se promueva la participación de sus usuarios dentro de la dinámica educativa, tanto en su propia aula como en la comunidad escolar. Esto ya sea con sus pares etarios, como con el personal docente y administrativo de la institución, en pro de derribar barreras de interacción y lograr el proceso de aprendizaje con mayor accesibilidad. En otras palabras, el uso de la tecnología se traduce en un ajuste razonable que permite la libre participación de todas las personas en los procesos educativos.

Por ejemplo, el campo de las tecnologías del habla, en el cual se desarrollan sistemas para potenciar la interacción humano-computadora (mediante sistemas de reconocimiento de voz y generación de habla artificial) ha venido realizando avances significativos en las últimas décadas. Estos avances se ven reflejados en una serie de tecnologías que forman parte del entorno de muchas personas, integradas en teléfonos inteligentes, sistemas de navegación, entre otras.

Desde sus inicios, se ha visto el potencial que tienen estas tecnologías para beneficiar a la población con discapacidad. Sin embargo, existen retos importantes para su plena incorporación en cada contexto, los cuales deben ser analizados en sus particularidades e implicaciones, además de solventar aún algunos problemas teóricos y de implementación. 
Como indica Cabero-Almenara (2008, p.21),

Las barreras con las que se encuentran las personas con diferentes tipos de discapacidad son de un amplio espectro, y van desde las arquitectónicas hasta las de comunicación, las referidas a los procesos de enseñanza-aprendizaje, las del imaginario social creado sobre ellas, hasta las psicológicas.

Esto es importante de ser destacado porque para derribar cada una de esas barreras se requiere, como bien señala Deliyore Vega, "un cambio actitudinal y cultural para promover los valores de empatía, altruismo y respeto a la otredad" (2018, p.45). Y es precisamente ese respeto a las diferencias, el que se debe generar cuando hablamos de las barreras de comunicación con las que se encuentra la población en condición de discapacidad, donde el uso de la tecnología puede llegar a suplir o potencializar la función comunicativa.

Morales indica que

(...) gran cantidad de niños y niñas, por diferentes razones, van retrasando sus habilidades lingüísticas, específicamente, en la emisión de la palabra hablada y en la estructuración de frases y oraciones para comunicarse, y permanecer en un estadio no verbal o no lingüístico que puede estar estancado o haber adquirido matices comunicativos bastante coordinados, sin recurrir al habla, por ende, requiere de un sistema de comunicación alternativo (Morales Rodríguez, 2016, p. 155).

Es por lo anterior que cobra una amplia relevancia el uso de estrategias novedosas, investigación en tecnologías y sistemas que permitan facilitar el acceso de la persona en condición de discapacidad a los contenidos del currículo educativo. Con esto, potencializar en la persona su capacidad de pensamiento, aprendizaje y participación en el contexto educativo y en cualquier contexto en que se desenvuelva, al permitir la interacción con sus compañeros etarios y sus docentes. De esta manera el uso de las tecnologías del habla en un sistema de comunicación aumentativo y alternativo (SAAC) no es solo una estrategia, sino un derecho para poner en ejercicio las capacidades de este grupo poblacional.

El objetivo de este ensayo es evidenciar la vinculación de las tecnologías del habla y la inclusión educativa, desde la definición conceptual de ambos términos hasta la aplicación de los mismos según la evidencia bibliográfica. Para esto se realiza una revisión de las tendencias actuales en estas áreas, así como los usos potenciales que puedan tener las 
nuevas para apoyar los procesos de inclusión educativa en personas en condición de discapacidad.

\section{La inclusión educativa y las tecnologías del habla}

La comunicación verbal es una de las habilidades que caracterizan a los seres humanos. A lo largo de la evolución se ha convertido en la vía de transferencia de información por excelencia, donde se pueden ver reflejados los pensamientos, gustos, desagrados y todas las opiniones que nos permiten una participación plena en el contexto social, comunitario y familiar.

No todas las personas tienen la capacidad de expresarse por medio de su propia voz debido a múltiples circunstancias, entre ellas condiciones de discapacidad que comprometen de forma parcial o total la producción de la voz hablada. Esto puede constituir un obstáculo para la incorporación de la persona a contextos sociales, incluyendo los entornos educativos tradicionales, donde la comunicación verbal es predominante.

Las tecnologías del habla han surgido como opciones de alta tecnología que replican la capacidad humana de comunicarse con el habla, la escucha y comprensión de mensajes verbales, para implementarse en dispositivos electrónicos. Para dar una visión más amplia de las tendencias y puntos de encuentro de la educación inclusiva y las tecnologías del habla, en las siguientes secciones se contextualizan y desarrollan de forma más amplia estos conceptos.

\subsection{Educación Inclusiva}

La inclusión en general es un concepto acuñado para referirse a la participación plena del ser humano en diferentes entornos. Se hace referencia a este concepto desde una amplia perspectiva que contempla a toda la diversidad relacionada a género, etnia, orientación sexual, condición migrante, condición socioeconómica y discapacidad. Según Echeita y Ainscow (2011, p. 32) "la inclusión ha de ser vista como una búsqueda constante de mejores maneras de responder a la diversidad del alumnado... se trata de aprender a vivir con la diferencia y a la vez de estudiar cómo podemos sacar partido a la diferencia".

Cuando se habla de inclusión en materia de educación, se hace referencia a todas aquellas estrategias que permitan a la diversidad del alumnado ser participantes activos de su proceso de enseñanza y aprendizaje, sin discriminación alguna por pertenecer a uno de los denominados grupos vulnerables, en este caso la población con discapacidad. 
Por su parte, el concepto de educación inclusiva entendido como una educación para todas las personas data de la década de 1990, cuando en la Declaración de Salamanca, organizada por el gobierno de España y la UNESCO, se señala el derecho a la educación de todos los niños y niñas. Esta educación debe darse en equidad de oportunidades, respetando las características, necesidades, intereses y capacidades particulares de cada uno, partiendo de una programación oportuna propuesta por el sistema educativo para garantizar el acceso y la participación del estudiantado (Echeita y Ainscow, 2011, p. 16). Como lo describe Meléndez "En el campo de la Educación, el concepto inclusión comienza a tener cada vez un sentido más propio, construido sobre la base de buenas ideas para justificar y ejercer el derecho a educarse por encima de las particularidades personales y culturales" (2004, p. 8).

Estas premisas vienen a contar con el sustento de la Convención de Derechos de la Persona con Discapacidad de Naciones Unidas, que fue ratificada por Costa Rica como Ley 8661 en el año 2008, la cual establece en su artículo 24 el derecho a la educación inclusiva, concepto que aún es un ideal, pero ni una realidad en la práctica docente del Sistema Educativo de nuestro país. En el artículo 2 de esta misma Convención, se reconoce como comunicación los medios de voz digitalizada, lo que nos lleva a considerarlos como un medio indispensable, en aquella población que los requiera, para garantizar su derecho a la participación en cualquier contexto, incluido el educativo. No obstante, pese a que han pasado ya más de diez años de su ratificación en Costa Rica, se sigue teniendo una deuda para con la población en condición de discapacidad, brecha que se amplía en el campo de la comunicación y el uso de la tecnología y más aún en la relación de estas mismas.

Pese a vivir en un mundo digitalizado en donde los recursos tecnológicos están cada vez más cercanos a la población estudiantil, el grupo de estudiantes con discapacidad se enfrentan a una barrera de acceso a la tecnología por diversas razones que van desde recursos económicos hasta desconocimiento de los dispositivos existentes para facilitar el acceso al currículo escolar. Sin lugar a dudas, esta población se vería favorecida con el uso de tecnologías que medien y promuevan procesos de enseñanza y aprendizaje sustentados en el DUA y en particular en el impacto positivo del uso de las tecnologías de la información y la comunicación (TIC) para maximizar el potencial del alumnado, en cuanto a habilidades como la de comunicación desde diversos formatos.

A pesar de las mencionadas barreras, se han dado algunos pasos para dar cabida en las aulas del sistema regular a la participación de población en condición de discapacidad. 
La diversidad de condiciones que puede presentar el alumnado invita al personal docente a su cargo a buscar variadas estrategias para poder desarrollar en conjunto con el niño o niña y su familia el proceso de enseñanza aprendizaje. No obstante, cuando la condición del estudiantado enfrenta al personal docente a una comunicación no verbal, el uso de un sistema de comunicación aumentativo y alternativo es requerido para mediar el proceso pedagógico.

Para comprender mejor el concepto se debe señalar que los sistemas aumentativos y alternativos de comunicación son aquellos que potencializan o sustituyen la voz del usuario, lo que permite definir la comunicación alternativa como "cualquier forma de comunicación distinta al habla y empleada por una persona en un contexto de comunicación cara a cara" (Warrick y Sociedad Internacional para la Comunicación Aumentativa y Alternativa, 2002, p. 95)

Estos sistemas pueden ser de bajo costo como pictogramas impresos o de alta tecnología como tabletas, teléfonos y dispositivos específicos en los que se utilicen voces artificiales que facilitan sin lugar a dudas la interacción comunicativa. No obstante, cabe aclarar que de las características de los sistemas llama la atención su versatilidad y amplitud para ser usados por una variedad de sectores ya que han sido clasificados según sus posibilidades de uso sin apoyo externo o con apoyo externo.

Esta categorización se vuelve de suma importancia para la planificación de prototipos que puedan solventar las necesidades de diversidad de usuarios que requieran de comunicación asistida o con apoyo la cual ha sido definida por varios autores como Tamarit desde 1989 como aquellos que requieren el uso de sistemas representacionales, mismos que pueden ser traducidos como herramientas, dispositivos o cualquier otro tipo de material más allá del propio cuerpo.

En ese sentido se puede afirmar que en Costa Rica, existen dispositivos comerciales de alta tecnología que facilitan la comunicación asistida o con apoyo. No obstante, el costo de los mismos, sus requerimientos por parte del usuario y de los mediadores comunicativos, así como su entrenamiento, han hecho que no sean dispositivos tan comúnmente usados para la población con discapacidad. Esto se vuelve controversial ya que al consultar los principios del diseño universal del aprendizaje, así como el objetivo cuatro dentro de los objetivos de desarrollo sostenible, que se enmarca en la agenda 2030 de la ONU, queda clara claro que la asequibilidad las tecnologías de apoyo como lo son las TIC y concretamente las tecnologías del habla deben ser asequibles para todas las personas que 
así lo requieran ya que esto promovería una educación inclusiva, equitativa y de calidad para todo el alumnado, pues es evidente que el usuario de un sistema alternativo de comunicación se ve beneficiado con un dispositivo de voz para su plena inclusión en cualquier contexto en que se desenvuelva.

\subsection{Tecnologías del habla}

Las tecnologías del habla reúnen una amplia gama de especialidades que analizan y procesan el habla humana para desarrollar sistemas de comunicación en ambos sentidos: la escucha y comprensión de mensajes hablados (reconocimiento de habla), y la emisión de mensajes con voces artificiales (conversión texto a voz, o síntesis de voz). Adicionalmente, en el procesamiento de la voz humana es posible reconocer patrones de interés para detectar emociones o alteraciones, lo cual es fuente de investigaciones actuales para integrarse a distintos entornos y apoyar sistemas de diagnóstico médico, aprendizaje de idiomas, entre otros.

Las dos áreas principales de las tecnologías del habla, el reconocimiento y la síntesis de voz, ofrecen una gran cantidad de posibilidades, algunas de las cuales son una realidad en diversos dispositivos y entornos en nuestros días. Adicionalmente, como indican Koon y de la Vega (2014, p. 3), desde sus inicios "se ha reconocido su potencial para integrarse a SAAC en beneficio de la población con discapacidad". En primer lugar, como indica Hawkridge, Vincent y Hales (2018, p.4), por "el aspecto de comunicación, del cual depende el proceso de educación".

Para establecer las capacidades y limitaciones que esta tecnología presenta en la actualidad para la inclusión educativa, se describirán con mayor detalle sus áreas de investigación más representativas. Es importante destacar que es un área en constante evolución, de la cual emergen constantemente nuevas subespecialidades producto de nuevas técnicas de desarrollo y nuevos entornos para la implementación de las aplicaciones.

Los primeros esfuerzos por generar hablan de forma artificial datan del siglo XVIII, donde investigadores como Von Kempelen desarrollaron sistemas mecánicos para generar sonidos vocálicos y algunas palabras, utilizando un sistema de fuelles y tubos flexibles que pretendían emular el aparato fonador humano (Schroeder, 1993). Los avances más significativos tuvieron que esperar hasta el siglo $\mathrm{XX}$, donde con ayuda de los sistemas electrónicos, en la primera mitad del siglo, y las computadoras en la segunda mitad, fue posible generar los primeros sistemas capaces de procesar señales de habla para 
reconocerlas como texto o comandos, y generar por su parte algunas palabras aisladas de habla artificial. Se pretendía generar palabras a partir de códigos y algoritmos, a diferencia de las palabras pregrabadas que simplemente vuelven a reproducir un contenido almacenado. Esto es importante, ya que es imposible pre-grabar todas las palabras que se utilizan en un idioma.

En nuestros días, el reconocimiento de habla es un área de activa investigación y desarrollo que utiliza algoritmos y sistemas cada vez más sofisticados. Su integración en dispositivos como computadoras y teléfonos celulares ha sido una realidad hasta épocas muy recientes debido a la complejidad del proceso de comunicación verbal y los distintos contextos donde se desarrolla (entornos con ruido, diversidad de acentos, géneros, vocabulario, edad). Existen aún problemas para esta dirección de comunicación sobre los que se debe avanzar para emular la capacidad humana de escucha y comprensión (Yu y Deng, 2016).

El reconocimiento de habla puede representarse como un sistema de búsqueda, comparación y evaluación de información sonora. En la Figura 1 se muestra un esquema de este proceso. En ésta, la palabra pronunciada se refiere a la vocalización de una palabra por una persona, la cual debe ser registrada a través de un micrófono para ser procesada por la computadora o dispositivo. La extracción de parámetros se refiere a la representación interna de la información que requiere la computadora. Usualmente se requieren complejos algoritmos de conversión de información para representar de forma numérica la voz.

Dentro del sistema deben estar almacenados parámetros de una gran cantidad de palabras y expresiones, para realizar comparaciones con los correspondientes al mensaje recibido y así poder tomar la decisión de establecer cuál palabra o frase fue emitida. Es importante recalcar que los sistemas de reconocimiento requieren que todos los sonidos y palabras de una lengua estén representados internamente, o bien contar con sistemas de actualización automática para incorporar cada vez más. Esto último ha sido posible recientemente gracias a la incorporación de inteligencia artificial, la cual puede ser entendida como el conjunto de teorías y sistemas que emulan las funciones de aprendizaje del cerebro humano, y que son capaces de ajustarse constantemente conforme reciben más información de su entorno.

Para poder resolver cada una de estas etapas, las tecnologías del habla en la actualidad se entienden como un área multidisciplinaria, ya que las mejoras en los sistemas de reconocimiento se requiere no sólo de técnicas avanzadas de programación o de 
algoritmos para el procesamiento de señales, sino de un adecuado conocimiento de los mecanismos físicos y sociales que permiten a la producción y la percepción del habla (Llisterri, 2003).

Todos estos procesos se esquematizan en la Figura 1, en la cual cada nivel que se recorre desde la palabra pronunciada por la persona (en el nivel superior) hasta la detección de la palabra por la computadora o sistema electrónico (nivel inferior) transita por una serie de representaciones internas y sistemas de clasificación que discriminan los sonidos emitidos para relacionarlas con las palabras existentes en determinado idioma.

Figura 1

Esquema del proceso de Reconocimiento Automático del Habla.

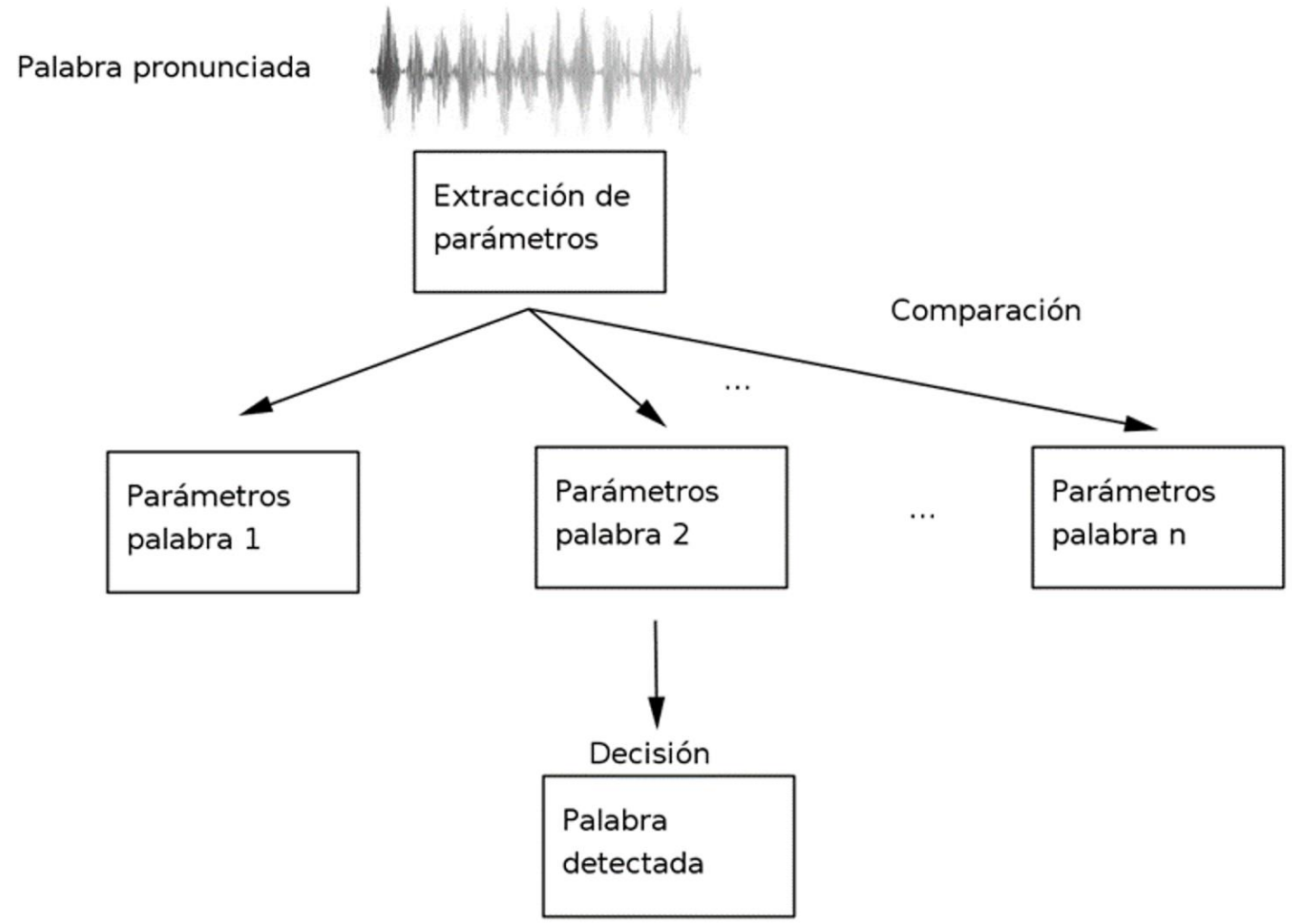

Fuente: Elaboración propia, 2019.

Por su parte, la síntesis de voz es el proceso de generar habla artificial inteligible a partir de un texto, para propiciar la interacción humano-computador. Este proceso conlleva una serie de etapas para analizar el texto y finalmente generar los sonidos que se deben producir para asemejar la pronunciación de una persona al leerlos. En este caso, "los 
mayores retos surgen al tratar de imitar las capacidades humanas de hablar con distintas emociones, con características únicas para cada persona, las cuales también conllevan información referente a edad, género y proveniencia" (Coto-Jiménez y Goddard-Close, 2016, p. 20).

El proceso de generar habla de forma artificial puede esquematizarse como en la Figura 2. En ésta, la palabra que se desea pronunciar debe analizarse e identificar los sonidos con los que se cuenta en una base de datos que pueden asemejarse más a aquellos que la constituyen. Estos sonidos se almacenan en forma de modelos matemáticos que los describen. Al identificar aquellos que sean más convenientes, se recopilan y se genera con ellos una onda de sonido que se escucha como habla.

En esta figura, el proceso parte de la representación interna más abstracta de la información, hasta la identificación de los sonidos particulares en sus modelos matemáticos, y llega, en la parte inferior, a la realidad física del sonido que se percibe como habla con un mensaje particular.

\section{Figura 2}

Esquema del proceso de Síntesis de voz.

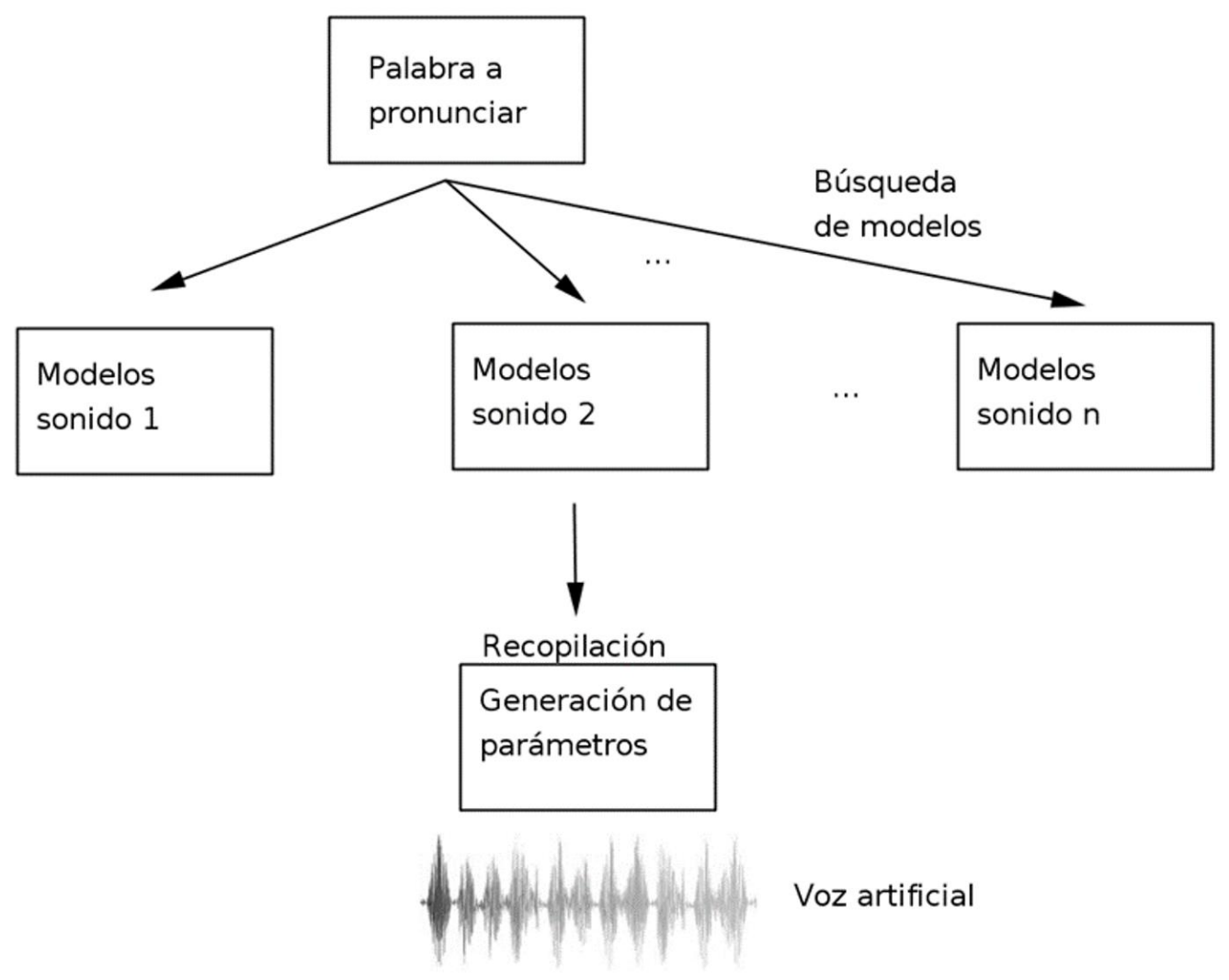

Fuente: Elaboración propia, 2019. 
Es importante recalcar que la producción de habla humana tiene como característica la variabilidad. Es decir, cada vez que se produce una palabra se hace con variaciones en el tono y la duración de los sonidos particulares, aún una misma persona. Cuando se desarrolla un sistema de síntesis de voz que no puede emular estas características, se percibe como un sistema robotizado y no es aceptado para su aplicación en todos los contextos posibles.

Las situaciones donde la síntesis de voz muestra sus ventajas son aquellas donde se tiene información a la que se debe acceder cuya naturaleza sea cambiante o no se puede predecir (tales como las direcciones, el contenido de Internet, descripción de entornos y contextos), o bien cuyo volumen de información no permite que un locutor humano lo grabe previamente. En el caso de las personas ciegas, esta tecnología ha sido empleada para tener acceso a información proveniente de Internet, de un documento escrito, o a un mensaje de correo, entre otros.

Uno de los aspectos del desarrollo de sistemas de producción artificial de habla que lo hace más complejo, es que cada sistema depende del idioma de procedencia y sus variantes, para lo cual es necesario adaptar los conceptos lingüísticos y programas de computadora involucrados. Esto es evidente si se considera que las técnicas recientes fueron inicialmente probadas en idiomas como el inglés y el japonés, a partir de las cuales se requiere hacer todo un proceso de generación de datos propios para que investigadores en cada país puedan reproducir los resultados en su idioma respectivo. Más aún si se considera que es deseable desarrollar voces que emulen particularidades como el género, edad, ritmo, entonación, acento, cadencia y emociones. Incluso "se investiga la generación artificial de otros aspectos como la risa" (El Haddad, Dupont, Urbain, y Dutoit, 2015, p. 1) y "elementos de duda y sonidos pausados propios de gran parte de la comunicación humana" (Nakanishi, Inoue, Nakamura, Takanashi y Kawahara 2019, p. 91).

\subsection{Pertinencia y aplicabilidad de las tecnologías del habla}

La diversidad de tecnologías disponibles para la educación inclusiva se muestra en la Tabla 1, donde se indican las potencialidades que presentan en procesos educativos a distintos niveles. 
Tabla 1

Recuento de tecnologías del habla con sus aplicaciones potenciales en educación inclusiva

\begin{tabular}{|c|c|c|}
\hline Tecnología & Descripción breve & Aplicación potencial \\
\hline $\begin{array}{l}\text { Reconocimiento } \\
\text { de voz }\end{array}$ & $\begin{array}{l}\text { Transcripción de } \\
\text { palabras habladas } \\
\text { a texto. }\end{array}$ & $\begin{array}{l}\text { Se puede utilizar para generar subtítulos en vídeos de } \\
\text { forma automática, o transcribir contenidos dados en una } \\
\text { clase (Ranchal, Taber-Doughty, Guo, Bain, Martin, } \\
\text { Robinson, y Duerstock, 2013). } \\
\text { Así mismo puede ser usada como control de mando por } \\
\text { usuarios con restricción de movilidad. }\end{array}$ \\
\hline Síntesis de voz & $\begin{array}{l}\text { Generación de } \\
\text { habla artificial en un } \\
\text { dispositivo de alta } \\
\text { tecnología. }\end{array}$ & $\begin{array}{l}\text { Dispositivo de comunicación a partir de texto, el cual es } \\
\text { pronunciado utilizando una interfaz, y de esta manera se } \\
\text { participa en conversaciones (Nguyen, 2018). } \\
\text { El cuál puede ser utilizado por usuarios no verbales o } \\
\text { con dificultades considerables para la producción oral } \\
\text { con diversas condiciones de discapacidad. }\end{array}$ \\
\hline $\begin{array}{l}\text { Reconocimiento } \\
\text { de hablante }\end{array}$ & $\begin{array}{l}\text { Identificación de } \\
\text { una persona en } \\
\text { particular a partir de } \\
\text { su voz, como } \\
\text { complemento al } \\
\text { reconocimiento de } \\
\text { habla. }\end{array}$ & $\begin{array}{l}\text { Se utiliza para acceder a contenido privado utilizando la } \\
\text { voz del usuario, o para discriminar la información } \\
\text { recibida y evitar un mal uso de sistemas (Hansen y } \\
\text { Hasan, 2015). En este sentido, se puede utilizar como un } \\
\text { complemento de las otras tecnologías del habla. }\end{array}$ \\
\hline $\begin{array}{l}\text { Reconocimiento } \\
\text { de emociones }\end{array}$ & $\begin{array}{l}\text { Detectar el estadio } \\
\text { emocional del } \\
\text { usuario a partir } \\
\text { solamente de su } \\
\text { voz, } \\
\text { complemento } r \text { al } \\
\text { reconocimiento de } \\
\text { habla. }\end{array}$ & $\begin{array}{l}\text { En sistemas de enseñanza automatizados, la detección } \\
\text { de la emoción presente es de importancia para adaptar } \\
\text { el contenido o alertar sobre posibles situaciones de } \\
\text { estrés o emociones que deban ser atendidas (Tickle, } \\
\text { Raghu, y Elshaw, 2013). } \\
\text { Por tanto, pueden ser de uso valioso para personas que } \\
\text { requieran apoyo para reconocer las emociones dentro de } \\
\text { un contexto de interacción social o cuando se esté } \\
\text { trabajando dicha temática dentro del entorno del aula. }\end{array}$ \\
\hline 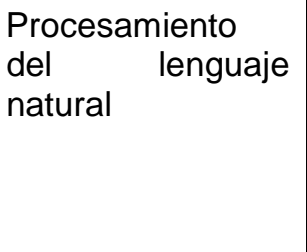 & $\begin{array}{l}\text { Interpretación de } \\
\text { los contenidos de } \\
\text { mensajes hablados } \\
\text { en lenguaje } \\
\text { cotidiano. }\end{array}$ & $\begin{array}{l}\text { Reconocer, procesar e interpretar el lenguaje en } \\
\text { cualquier contexto y nivel de desarrollo educativo. Esto } \\
\text { es de importancia para poder recibir mensajes en } \\
\text { cualquier variante del idioma y para guiar a los usuarios } \\
\text { hacia formas de expresión más desarrolladas (Allen, } \\
\text { Snow, y McNamara, 2015). }\end{array}$ \\
\hline
\end{tabular}

Fuente: Elaboración propia, 2019.

Un ejemplo concreto de sistemas que implementan estas tecnologías de forma eficiente son los asistentes personales virtuales, en los cuales el habla producida por una persona es registrada a través de un micrófono y transcrita dentro de una computadora 0 
dispositivo para poder ser mostrada y almacenada en formato texto, a la cual se responde usualmente con un mensaje de habla artificial. En la actualidad es posible observar un uso cotidiano de esta tecnología en teléfonos celulares, para realizar consultas y búsquedas en Internet.

De esta manera, es posible plantear la extensión de estas posibilidades para permitir el acceso a la información presentada durante un discurso, explicación u otra presentación verbal tanto a personas ciegas (para ser leído posteriormente por un lector de pantalla) como a personas sordas, y de esta manera facilitarle el acceso a la información. Si bien el uso de estas posibilidades se ha vuelto cotidiano, existen retos significativos para hacerla una realidad en todos los contextos donde se necesita, producto de las variadas condiciones ambientales y de uso que afectan el proceso.

Por ejemplo, el registro de la voz de un profesor o ponente en un aula puede verse afectado cuando la persona se desplaza o habla en distintas direcciones. En el micrófono que capta la señal, pueden aparecer ecos y disminuciones en la señal que afectan la transcripción automática. Algunas soluciones pueden establecerse en términos de utilizar varios micrófonos, o aplicar algoritmos de mejora de señal ante estos cambios.

En términos más generales, cuando consideramos la pertinencia y el impacto del uso de las tecnologías del habla en el diseño de tecnología al servicio de la comunicación para personas en condición de discapacidad, surge la consulta lógica de a cuál población en específico se estaría beneficiando.

Lo anterior llama al análisis porque el conglomerado poblacional que se reúne bajo el concepto de personas con discapacidad es muy amplio y diverso, por tanto sostener que un sistema de comunicación aumentativo y alternativo sustentado en el uso de las tecnologías del habla puede ser supeditado a una sola condición de discapacidad es reducir su potencial. Esto bajo la premisa de que el mismo puede y debe ser utilizado por aquellos usuarios que así lo requieran y de esta forma ampliar sus posibilidades de uso para todas aquellas personas que presentan dificultad o imposibilidad para desarrollar un lenguaje oral inteligible.

Por lo anterior, el uso de este tipo de recurso tecnológico puede ser un apoyo para población en condición de discapacidad motora, discapacidad sensorial, autismo, discapacidad intelectual o discapacidad múltiple. Cada una de estas condiciones tendrá sus necesidades comunicativas y en sus procesos de aprendizaje, para lo cual las particularidades deben tomarse en cuenta. 
En esta línea, debido a la variabilidad de formas comunicativas que pueden presentar las personas con discapacidad, la Dra. Charity Rowland presentó en 1990 una propuesta para la comprensión de los diversos niveles de comunicación del ser humano que van desde las conductas no intencionales hasta el uso del lenguaje en siete niveles de comunicación, en formato de una matriz, organizada en cuatro razones principales para comunicarse: para reusar cosas que no queremos; para obtener cosas que sí queremos; para entrar en relación social; y para proporcionar o buscar información (Rowland, 2013). En la Tabla 2 se muestra cómo las tecnologías del habla pueden ser usadas específicamente para el desarrollo de habilidades de los últimos tres niveles de la matriz de comunicación y que son totalmente aplicables al trabajo de aula y otros contextos de la vida del estudiantado con discapacidad.

Tabla 2

Usos de las tecnologías del habla según niveles de comunicación

\begin{tabular}{|l|l|l|}
\hline $\begin{array}{c}\text { Nivel primario de } \\
\text { funcionamiento } \\
\text { comunicativo }\end{array}$ & \multicolumn{1}{|c|}{$\begin{array}{c}\text { Meta general del } \\
\text { nivel }\end{array}$} & \multicolumn{1}{|c|}{ Tecnologías del habla } \\
\hline Símbolos Concretos & $\begin{array}{l}\text { Enseñar la } \\
\text { correspondencia uno a } \\
\text { uno entre símbolos } \\
\text { abstractos } \\
\text { referentes. }\end{array}$ & $\begin{array}{l}\text { La síntesis de voz puede ser aplicada a diversos } \\
\text { dispositivos de causa efecto como juguetes y } \\
\text { dispositivos que otorgan voz para nombrar fotos y } \\
\text { pictogramas. }\end{array}$ \\
\hline Símbolos Abstractos & $\begin{array}{l}\text { Enseñar la } \\
\text { combinación de } \\
\text { símbolos en dos a tres } \\
\text { expresiones simbólicas }\end{array}$ & $\begin{array}{l}\text { En este nivel el uso del reconocimiento de } \\
\text { emociones y la síntesis de voz pueden ser de gran } \\
\text { utilidad identificar identificar y combinar 2 } 3 \\
\text { imágenes con su respectivo sonido o nombre. }\end{array}$ \\
\hline Lenguaje & $\begin{array}{l}\text { Expandir habilidades } \\
\text { semánticas } \\
\text { sintácticas }\end{array}$ & $\begin{array}{l}\text { Para este nivel comunicativo se amplían las } \\
\text { propuestas del nivel de símbolos abstractos con la } \\
\text { estructuración de frase formadas con la } \\
\text { combinación de imágenes, pictogramas y voz } \\
\text { artificial. }\end{array}$ \\
\hline Lenguaje & $\begin{array}{l}\text { Desarrollar habilidades } \\
\text { pragmáticas }\end{array}$ & $\begin{array}{l}\text { Para este nivel comunicativo donde se pretende el } \\
\text { uso de una combinación de las habilidades } \\
\text { anteriores se puede hacer uso de todos los } \\
\text { recursos de las tecnologías del habla, incluido el } \\
\text { procesamiento de lenguaje natural con el } \\
\text { propósito de generar oraciones y comunicaciones } \\
\text { de mayor extensión }\end{array}$ \\
\hline
\end{tabular}

Fuente: Elaboración propia, con información adaptada de Rowland (2013, p. 40). 
Por su parte y como se ejemplifica previamente, cada condición de uso puede presentar retos significativos, donde cada salón de clase y entorno tienen particularidades que requieran adaptar la tecnología a estos contextos. De esta manera los retos son permanentes y la necesidad de contar con suficientes personas capacitadas para abordarlos es parte de los requerimientos indispensables para que la aplicabilidad sea general, y los beneficios puedan extenderse.

\section{Consideraciones para la implementación de sistemas tecnológicos de comunicación}

Dada la complejidad propia del desarrollo de las tecnologías del habla, y sus necesidades de adaptación a cada caso particular, para llevar a la realidad su impacto favorable a la inclusión educativa es importante considerar aspectos como la participación directa e involucramiento de las personas con discapacidad, ya que esto permite detectar aspectos que no se encuentran dentro de las experiencias y visión de los desarrolladores. Más aún, permite realmente definir como punto de partida cuáles son las necesidades reales y los requerimientos como punto de partida.

El uso de tecnologías el habla en SAAC, pese a ser un recurso de vanguardia, presenta también retos para el usuario y los mediadores comunicativos, ya que aproximadamente entre el $24 \%$ y el $56 \%$, los usuarios de tecnologías de asistencia dejan de utilizar estas tecnologías, cuando las mismas no calzan bien con las preferencias personales o con los hábitos rutinarios de la persona según indica Shinohara (2017). Esto se ve se apoyado por Carmien (2015, p. 5), quien reporta "un porcentaje estimado de hasta el $70 \%$ entre todos los usuarios a largo plazo". Las razones van desde las dificultades para configurar y modificar los dispositivos hasta los cambios en las necesidades de los usuarios.

Recientemente se presentó el concepto de diseño socialmente accesible para las tecnologías de asistencia para personas con discapacidad, en el cual se debe tomar en cuenta la aceptación de los diseños en el entorno donde el usuario se desarrolle. Según Shinohara (2017) es importante abordar el problema de la estigmatización y negatividad que existe en la implementación final de algunos productos. Para esto se propone la accesibilidad social como una nueva característica de las tecnologías para incluir situaciones sociales de su uso. Esto afianza la instauración del modelo social con enfoque de derechos humanos al fomentar la inclusión en diversos ambientes donde cada individuo puede desenvolverse. 
Ha sido observado cómo factores sociales influencian la manera en que las personas con discapacidad usan los dispositivos tecnológicos, frecuentemente llevamos por concepciones erróneas de las tecnologías de asistencia y la forma de usarlas. Al contrario de la mayoría de tecnologías personales, que son discrecionales y en las cuales existen varias opciones, las de asistencia usualmente tienen opciones limitadas, propietarias.

El sentirse estigmatizados es una de las razones por las cuales algunas personas usuarias abandonan la idea de utilizarlos, por ejemplo, ante dispositivos que son muy evidentes o que se perciban como extraños ante contextos cotidianos. Por esto es importante establecer criterios sociales, además de las funciones del o los dispositivos seleccionados por la persona usuaria, su familia y equipo de apoyo pedagógico y terapéutico, para el éxito de la implementación de tecnología en la discapacidad.

Los tres criterios propuestos según Shinohara (2017) para el diseño de tecnologías de asistencia, las cuales pueden contemplar sistemas de reconocimiento y síntesis de voz son:

(a) que el proceso de diseño incluya a personas con y sin discapacidad como los usuarios destino del desarrollo; (b) el diseño debe incorporar situaciones sociales de uso, tanto como situaciones funcionales. Se ha observado cómo algunos diseños funcionales no se consideran socialmente aceptables, lo cual lleva a que finalmente no sean utilizados; (c) los diseñadores deben considerar diferentes perspectivas y reflejar cómo los productos podrían influenciar, o ser influenciados por situaciones sociales (p. 181).

Lo anterior incluye aspectos como la participación familiar, la valoración del entorno y las particularidades de cada individuo.

Es conocido el hecho de que cada comunidad tiene una forma especial de comunicarse verbalmente, reflejada en su lengua, acento particular, vocabulario y modismos. Estos elementos forman parte de su identidad, y en términos del desarrollo de tecnologías, deben tomarse en cuenta para que los resultados puedan ser aceptados de forma más directa por el usuario y su entorno.

Uno de los casos más claros es el de niños. Diversos estudios han mostrado que los sistemas de reconocimiento de habla se ven degradados en su eficiencia cuando se trata de procesar voces de menores de edad. Por su parte, la producción artificial de voz de niños es un tema poco abordado en los diferentes grupos alrededor del mundo. Esto se debe a las dificultades propias del proceso de grabar suficiente información de los niños, con el reto 
adicional de cubrir una cantidad de palabras considerables, que no forman parte del vocabulario usual en este grupo de edad.

Como indican Guenaga, Barbier y Eguiluz (2017, p. 156):

Para garantizar el acceso a cualquier recurso tecnológico a todas las personas, incluidas las que sufren algún tipo de discapacidad, no es suficiente con desarrollar tecnologías asistidas adecuadas para cada tipo de discapacidad. También es necesario que los propios recursos tecnológicos están diseñados para que puedan ser accedidos a través de estas ayudas técnicas.

Por ejemplo, en el caso de un sistema que pueda generar voz de forma artificial para poder acceder a información de Internet, se tendría que ir entonces más allá de un lector de texto que pronuncie las palabras contenidas en un determinado sitio web. Sería necesario que el propio sitio web estuviera diseñado para que toda la información contenida, incluyendo imágenes, videos, figuras y texto matemático, tuviera, además del contenido en sí, una descripción o transcripción sugerida para ser leída por el sistema. De esta manera se podría generar en formato de voz una descripción de lo que una imagen muestra, lo que esquematiza una figura o lo que dice una fórmula matemática. En la misma línea, las personas autoras indican la necesidad "de continuar esfuerzos por generar una mayor cantidad de recursos y posibilidades" (Guenaga, Barbier y Eguiluz, 2017, p. 157).

No son muchas las interfaces que se manejan a través del habla, o por reconocimiento de voz, pero sí hay líneas de investigación y cada vez más productos comerciales que la utilizan. Las personas con dificultad en el habla encontrarán dificultades a la hora de manejar este tipo de interfaces, si no se les proporcionan otras alternativas.

Davis (2012) en su investigación establece algunas visiones expresadas por usuarios de tecnologías de asistencia en instituciones universitarias de Estados Unidos, en las cuales se ha difundido su uso. En dicho estudio, los usuarios establecen la importancia que tendría la generación automática de notas de clase a partir del habla del profesorado, utilizando tecnologías de reconocimiento de voz, mientras dictan la lección. Esto en formatos que puedan ser trasladados directamente a diversos dispositivos (computadoras, celulares, tabletas).

\section{Síntesis y reflexiones finales}

Para que un sistema de reconocimiento de habla pueda asemejarse a la capacidad humana de escucha y comprensión debe contar con características que le permitan procesar 
habla producida por cualquier persona, a diferentes velocidades y en entornos variados y con ruidos ambientes. Por ejemplo, al pensar en un sistema de transcripción de habla, con el cual se podría hacer accesible una clase magistral regular, se debe contemplar que el entorno tiene condiciones que hacen degradar el proceso de reconocimiento, tales como ecos, sonidos ambiente, otras voces y palabras técnicas de uso especializado.

Al considerar sistemas que identifiquen mensajes emitidos en forma de habla también se deben contemplar aspectos clave que forman parte del proceso de comunicación humana, y que se encuentran actualmente como problemas abiertos en el desarrollo de esta tecnología, sobre los cuales se están aplicando algoritmos cada vez más sofisticados en la investigación.

Algunos de estos aspectos son: (a) cuando se tienen varias personas hablando, identificar quién emite el mensaje; (b) identificar la emoción con la cual se está hablando; (c) identificar intenciones dependientes del contexto, como el sarcasmo; (d) captar mensajes con condiciones cambiantes (la persona camina, sale, hay lluvia, ruido del tráfico, etc.).

Cada uno de estos aspectos es desarrollado por grupos de investigadores alrededor del mundo, y se requiere un gran esfuerzo de integración para llevar a la realidad sistemas que apoyen los procesos de inclusión educativa y que sean accesibles en precio y características como portabilidad y robustez.

Por su parte, un sistema de síntesis de voz que le permita una persona emitir con ayuda de un dispositivo mensajes con habla artificial, debe prestar atención a aspectos prosódicos, relacionados con las características de entonación y duración que se producen a lo largo de las frases, gracias a las cuales distinguimos si se trata de una pregunta, una exclamación o se ha llegado a una pausa ortográfica, como un punto.

En la emisión del habla intervienen una gran cantidad de órganos del aparato fonoarticulador, además de aspectos evolutivos y culturales, lo que hace que el proceso y su emulación artificial sean muy complejos. Los objetivos que se persiguen en la actualidad en la investigación en esta área son la flexibilidad y la naturalidad, es decir, un parecido "suficiente con las características humanas que permitan extender la utilización a más usuarios y las posibilidades de más aplicaciones" (Coto-Jiménez, Goddard-Close y MartínezLicona, 2015, p. 32).

Así mismo desde el punto de vista de la persona en condición de discapacidad deben valorarse aspectos como los puntos de acceso para accionar estos sistemas, porque no podemos pretender que todo sirva para todos. 
Adicionalmente, es deseable que cada persona que requiera de un dispositivo de generación de voz pueda contar con una voz única, que esté acorde con su edad, género y el acento propio de su área geográfica. El contar con estas características podría no solo permitir eliminar barreras de comunicación, y fomentar la inclusión de personas con discapacidad en centros educativos, sino también potencializar en ese individuo el sano desarrollo de aspectos de personalidad y autoestima.

Otro de los importantes retos en el uso de las tecnologías del habla para usuarios en condición de discapacidad es la capacitación en el uso del sistema. No solamente al usuario, sino también a los mediadores comunicativos dentro del entorno familiar y el educativo. La importancia de que los y las educadores conozcan sobre estas tecnologías permitirá la comprensión de las mismas y su uso en el quehacer pedagógico para acercar al estudiantado a una oportuna participación educativa que le provea de los procesos de aprendizaje según sus características y habilidades.

Todas las consideraciones expuestas anteriormente brindan un panorama lleno de posibilidades de aplicación de la tecnología del habla para impactar la calidad de vida de la población con discapacidad. Sin embargo, la cantidad de retos también es muy grande. Se han señalado aspectos de mucha importancia, como involucrar a las propias personas con discapacidad y a sus familias para tener éxito en implementar los productos tecnológicos. $Y$ desde el punto de vista del desarrollo, es vital contar con equipos multidisciplinarios al menos de áreas relacionados con la tecnología, la educación especial, la orientación, el trabajo social, la psicología y la fisioterapia.

De esta manera, se pueden establecer pautas, procesos y productos arraigados verdaderamente en las necesidades y particularidades, y que puedan ser integrados plenamente en beneficio de la población con discapacidad. Así, el impacto en la inclusión educativa y el respeto a los derechos humanos puede contar con mejores garantías de realizarse con un aprovechamiento real y a largo plazo.

\section{Agradecimientos}

El presente trabajo ha sido desarrollado gracias al apoyo de la Universidad de Costa Rica, proyecto ED-3416.

\section{Referencias}

Ley 8661 Aprobación de Convención de Derechos de la Persona con Discapacidad. (2008). San José, Costa Rica. Asamblea Legislativa. 
Alba-Pastor, Carmen. (2012). Aportaciones del Diseño Universal para el Aprendizaje y de los materiales digitales en el logro de una enseñanza accesible. Recuperado de http://diversidad. murciaeduca. es/publicaciones/dea2012/docs/calba. pdf.

Allen, Laura, Snow, Erica y McNamara, Danielle. (2015). Are you reading my mind?: modeling students' reading comprehension skills with natural language processing techniques. In Proceedings of the fifth international conference on learning analytics and knowledge (pp. 246-254). New York: ACM.

Cabero-Almenara, Julio. (2008). TICs para la igualdad: la brecha digital en la discapacidad. Anales de la Universidad Metropolitana, 8(2), 15-43.

Carmien, Stefan. (2015). Assistive Technology Design for Intelligence Augmentation. Synthesis Lectures on Assistive, Rehabilitative, and Health-preserving Technologies, 5(2), 1-171.

Coto-Jiménez, Marvin, Goddard-Close, John. y Martínez-Licona, Fabiola. (2015) Producción artificial de habla: Síntesis de voz. Komputer Sapiens. 7, 6-10. Recuperado de http://smia.mx/komputersapiens/download.php?file=ks73 8.05MB extensa.pdf

Coto-Jiménez, Marvin. y Goddard-Close, John. (2016). Speech Synthesis Based on Hidden Markov Models and Deep Learning. Research in Computing Science, 112, 19-28.

Davis, Jeffrey. (2012). Perceptions of disability-services administrators about assistive technology at postsecondary institutions. Barry University-Adrian Dominican School of Education, Miami, Estados Unidos.

Decreto Ejecutivo №40955 - MEP del 19 de marzo. (2018). Publicado en La Gaceta № 59 del 19 de marzo. San José, Costa Rica: Imprenta Nacional.

Deliyore Vega, Rocío. (2018). Comunicación alternativa y aumentativa: Acciones y reflexiones para romper el silencio en las aulas. San José, Costa Rica: INIE-UCR.

Echeita, Gerardo. y Ainscow, Mel. (2011). La educación inclusiva como derecho: marco de referencia y pautas de acción para el desarrollo de una revolución pendiente. Tejuelo: Revista de Didáctica de la Lengua y la Literatura, 12, 26 - 46 Recuperado de http://iesgtballester.juntaextremadura.net/web/profesores/tejuelo/vinculos/articulos/r12/ 03.pd

El Haddad, Kevin., Dupont, Stéphan., Urbain, Jérome., y Dutoit, Thierry. (2015, April). Speech-laughs: an HMM-based approach for amused speech synthesis. In 2015 IEEE International Conference on Acoustics, Speech and Signal Processing (ICASSP). Queensland, Australia: IEEE 
Guenaga, María Luz., Barbier, Ander. y Eguiluz, Andoni. (2017). La accesibilidad y las tecnologías en la información y la comunicación. TRANS. Revista de traductología, (11), 155-169.

Hansen, John. y Hasan, Taufiq. (2015). Speaker recognition by machines and humans: A tutorial review. IEEE Signal processing magazine, 32(6), 74-99.

Hawkridge, David., Vincent, Tom., y Hales, Gerald. (2018). New information technology in the education of disabled children and adults. Routledge: London, England.

Koon, Ricardo, y de la Vega, María Eugenia. (2014). El impacto tecnológico en las personas con discapacidad.

Llisterri, Joaquim. (2003). Las tecnologías del habla: Entre la ingeniería y la lingüística. En Actas del Congreso Internacional "La Ciencia ante el Público. Cultura humanística y desarrollo científico y tecnológico", Universidad de Salamanca, Instituto Universitario de Estudios de la Ciencia y Tecnología (pp. 44-67). Salamanca, España.

Meléndez, Lady. (2004). Diversidad y equidad: Paradigma Educativo Urgente para la Costa Rica en vías de desarrollo. Revista parlamentaria, 12(2), 81-96.

Ministerio de Educación Pública de Costa Rica. (2018). Líneas de acción para los Servicios de Apoyo Educativo que se brindan desde la Educación Preescolar y en Primero y Segundo Ciclos de la Educación General Básica. Recuperado de http://www.drea.co.cr/sites/default/files/Contenido/L\%C3\%ADneas\%20Acci\%C3\%B3n \%20Apoyos\%20en\%20Preescolar\%20y\%20en\%201\%20y\%20I1\%20Ciclos\%202018.pdf

Morales Rodríguez, Maribel. (2016) De los sonidos a las palabras: Métodos y técnicas para la estimulación del lenguaje oral 1. San José, Costa Rica. Editorial EUNED

Nakanishi, Ryosuke, Inoue, Koj., Nakamura, Shizuka, Takanashi, Katsuya, y Kawahara, Tatsuya. (2019). Generating fillers based on dialog act pairs for smooth turn-taking by humanoid robot. In 9th International Workshop on Spoken Dialogue System Technology (pp. 91-101), Springer, Singapore.

Nguyen, Duy (2018). Initial Designs for Improving Conversations for People Using Speech Synthesizers. Oregon State University, United States of America.

Ranchal, Rohit., Taber-Doughty, Teresa., Guo, Yiren., Bain, Keith., Martin, Heather., Robinson, Paul., y Duerstock, Bradley. (2013). Using speech recognition for real-time captioning and lecture transcription in the classroom. IEEE Transactions on Learning Technologies, 6(4), 299-311.

Rowland, Charity. (2013). Handbook: Online Communication Matrix. Oregon Health \& Science University. United States of America. Recuperado de: http://www.communicationmatrix.org/uploads/pdfs/handbook.pdf

Schroeder, Manfred. (1993). A brief history of synthetic speech. Speech Communication, 13(1-2), 231-237. 
Shinohara, Kristen. (2017). Design for Social Accessibility: Incorporating Social Factors in the Design of Accessible Technologies (Doctoral dissertation). Recuperado de https://search-proquest-

com.ezproxy.sibdi.ucr.ac.cr/docview/1942838390?accountid=28692

Tickle, A., Raghu, S., y Elshaw, M. (2013). Emotional recognition from the speech signal for a virtual education agent. Journal of Physics: Conference Series, 450(1), 1-6.

Warrick, Anne., y Sociedad Internacional para la Comunicación Aumentativa y Alternativa. (2002). Comunicación sin habla: comunicación aumentativa y alternativa alrededor del mundo. CEAPAT.

Yu, Dong. y Deng, Li. (2016). Automatic Speech Recognition. Londres, Reino Unido: Springer London Limited. 
Revista indizada en

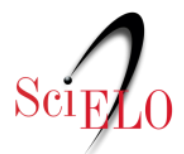

redalyc latindex

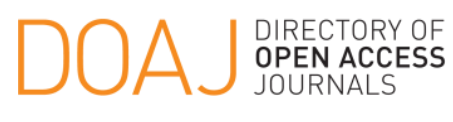

Distribuida en las bases de datos:

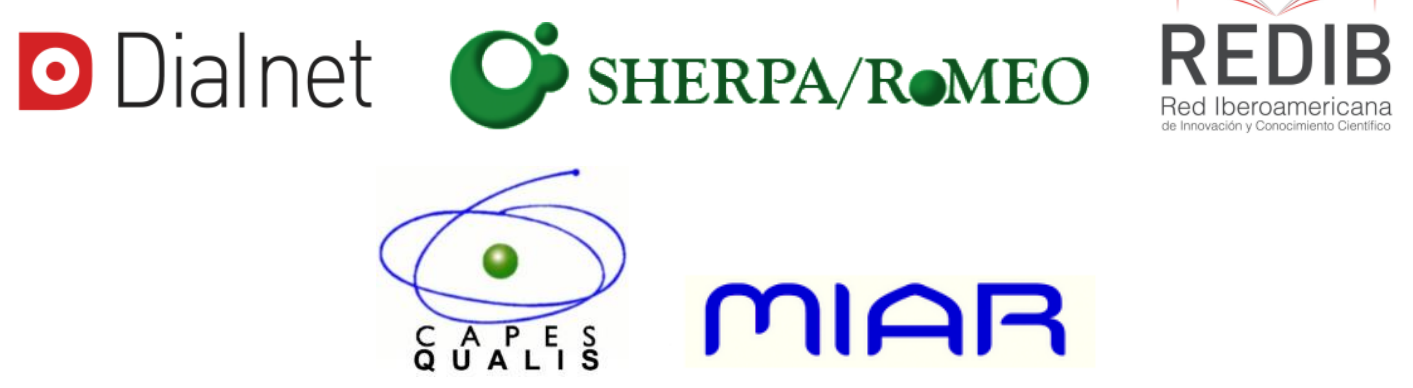

